\title{
Reduction of individual sampling rates by generalizing quadrature methods of separate interpolation
}

\author{
Aníbal R. Figueiras-Vidal and José B. Marino-Acebal \\ ETSI Telecomunicación, Cdad. Universitaria, Madrid 3, Spain
}

Miguel A. Lagunas-Hernández and Ramón García-Gómez

ETSI Telecomunicación, Jorge Girona Salgado s/n, Barcelona 34, Spain

(Received 2 December 1980; accepted for publication 13 July 1981)

\begin{abstract}
The limitations of sampling a bandpass signal at the (on the average) Nyquist rate and the possibility of equivalent low pass processing have stimulated research toward obtaining a separate interpolation for the inphase and quadrature components of the signal. Known results are to sample at a half the Nyquist rate of these in-phase and quadrature components, or, under Brown's condition, $\omega_{0}=k \sigma / 2$ (where $\omega_{0}$ and $\sigma$ are the center angular frequency and the angular bandwidth of the signal, respectively, and $k$ is an integer), to sample at the same rate the signal and its Hilbert transform or the signal and the signal delayed $\pi / 2 \omega_{0}$. Grace and Pitt [J. Acoust. Soc. Am. 44, 1453 (1968)] and Brown [IEEE Trans. Aerosp. Electron. Syst. AES-15, 366 (1979); J. Acoust. Soc. Am. 67, 1959 (1980)] have worked in the last direction, that has been generalized by the authors [Proc. First European Conf. Signal Proc., 197, Lausanne (1980)] to consider two general linear functionals of the signal. In this paper, the possibility of a further reduction of the sampling rate of the functionals maintaining a separate interpolation is shown: if we consider $2 N$ linear functionals (with a certain restriction) and $\omega_{0}=k \sigma / 2 N$ (generalized Brown's condition), an interpolation is possible from samples taken at $1 / 2 N$ the Nyquist rate, and separation is obtained when $N$ functionals have identically zero quadrature components of their impulse responses and the impulse responses of the other $N$ functionals do not show inphase components. Minimum oversampling rates required when generalized Brown's condition fails are also discussed.
\end{abstract}

PACS numbers: $43.60 . \mathrm{Cg}, 43.60 . \mathrm{Gk}$

\section{INTRODUCTION}

To recover a deterministic, real, finite energy, bandlimited bandpass signal $x(t)$ from its samples taken at the Nyquist rate $(\sigma / \pi$ samples/s, where $\sigma \mathrm{rad} / \mathrm{s}$ is the angular bandwidth of the signal) is only possible when the center angular frequency of $x(t), \omega_{0}$, equals $k \sigma / 2, k$ an odd integer. ${ }^{1}$ This limitation and the possibility of equivalent lowpass processing ${ }^{2}$ with the in-phase and quadrature components of $x(t)$, i.e., $p_{x}(t)$ and $q_{x}(t)$ in

$$
x(t)=p_{x}(t) \cos \omega_{0} t-q_{x}(t) \sin \omega_{0} t,
$$

respectively, have prompter research on sampling signals associated with $x(t)$ which will also allow a separate interpolation for $p_{x}(t)$ and $q_{x}(t)$, maintaining simultaneously the theor etically minimum (average) sampling rate $\sigma / \pi$.

Besides the obvious alternative of sampling the inphase and quadrature components of $x(t)$ at $\sigma / 2 \pi$, it is well known that we can also sample at $\sigma / 2 \pi$ both $x(t)$ and its Hilbert transform $\hat{x}(t)$ or $x(t)$ and $x\left(t-\pi / 2 \omega_{0}\right)$, obtaining a separate interpolation when $\omega_{0}=k \sigma / 2, k$ a positive integer..$^{1,3-5}$ The last equality will be indicated as Brown's condition.

These possibilities have been generalized recently ${ }^{6.7}$ to consider sampling the outputs of two linear time-invariant systems driven by $x(t)$ instead of $x(t)$ and $\hat{x}(t)$ or $x(t)$ and $x\left(t-\pi / 2 \omega_{0}\right)$. Under Brown's condition, we will arrive at a separate interpolation formula if the impulse response of one system has an identically zero quadrature component and the impulse response of the other shows an identically zero in-phase component.

In the present paper, we introduce a generalization of the above methods, considering $2 \mathrm{~N}$ linear time-invariant systems excited by $x(t)$, in order to allow further reductions of the sampling rate applied to each output, and imposing on the corresponding transfer functions conditions to obtain a separate interpolation. This procedure can be viewed as an useful particularization of a general multichannel bandpass sampling theorem proposed by Brown following two approaches: extending Papoulis' lowpass formulation ${ }^{8-10}$ for bandpass signals, ${ }^{11}$ and similarly extending a direct formulation ${ }^{12}$ to bandpass waveforms. ${ }^{13}$

\section{A GENERAL INTERPOLATION FORMULA}

We will follow a simple modification of Papoulis' generalized sampling expansion, ${ }^{8-10}$ that is applicable to lowpass functions.

Let $\left\{H_{i}(\omega)\right\}_{i=1}^{2 N}$ be the transfer functions of the $2 N$ linear time-invariant systems considered; we assume real systems, which implies Hermitian $H_{i}(\omega)$ :

$$
H_{i}(\omega)=H_{i}^{*}(-\omega), \quad i=1, \ldots, 2 N \text {. }
$$

We form the system of $2 N$ equations

$$
\begin{aligned}
& \sum_{i=1}^{2 N} H_{i}(\omega+r c) Y_{i}(\omega, t)=\exp (j r c t), \quad r=0, \ldots, N-1 \\
& \sum_{i=1}^{2 N} H_{i}\left(\omega+2 \omega_{0}+r c\right) Y_{i}(\omega, t) \\
& \quad=\exp \left[j\left(2 \omega_{0}+r c\right) t\right], \quad r=0, \ldots, N-1,
\end{aligned}
$$

where $c=\sigma / N$, and we assume that (3) possesses a solution $\left\{Y_{i}(\omega, t)\right\}_{i=1}^{2 N}$ in $\left(-\omega_{0}-\sigma / 2,-\omega_{0}-\sigma / 2+c\right)$; these re- 
strictions on the transfer functions constitute an "independence" condition.

The above system is a special case of the system used in Brown's earlier paper. ${ }^{11}$ This restriction and the generalized Brown's condition that follows are necessary to allow a separate interpolation, since, under general conditions, it is not possible to obtain it since the interpolation functions yield "asymmetrical" spectra about $\pm \omega_{0}$, that cause the in-phase and quadrature components to be different from zero. These "asymmetrical" spectra show discontinuities in their transfer functions. 6.7

$$
\begin{aligned}
& \text { If } 2 \omega_{0}=k c, \text { i.e., } \\
& \omega_{0}=k \sigma / 2 N,
\end{aligned}
$$

$k$ a positive integer, it will be easy to see that each $Y_{i}(\omega, t)$ is periodic with a period $T=2 \pi / c=2 \pi N / \sigma$. We will work under this restriction, that we designate as "generalized Brown's condition."

Each product $Y_{i}(\omega, t) \exp (j \omega t)$ can be expanded in a Fourier series in $\left(-\omega_{0}-\sigma / 2,-\omega_{0}-\sigma / 2+c\right)$ :

$$
Y_{i}(\omega, t) \exp (j \omega t)=\sum_{n=-\infty}^{\infty} y_{i, n}(t) \exp (j n T \omega),
$$

where

$$
\begin{aligned}
y_{i, n}(t) & =\frac{1}{c} \int_{-\omega_{0}-\sigma / 2}^{-\omega_{0}-\sigma / 2+c} Y_{i}(\omega, t) \exp [j \omega(t-n T)] d \omega \\
& =y_{i, 0}(t-n T) .
\end{aligned}
$$

Then

$$
Y_{i}(\omega, t) \exp (j \omega t)=\sum_{n=-\infty}^{\infty} y_{i, 0}(t-n T) \exp (j n T \omega)
$$

and, from the first equation of (3) multiplied by $\exp (j \omega t)$ and (7), we obtain

$$
\exp (j \omega t)=\sum_{i=1}^{2 \pi} H_{i}(\omega) \sum_{n=-\infty}^{\infty} y_{i, 0}(t-n T) \exp (j n T \omega)
$$

in $\left(-\omega_{0}-\sigma / 2,-\omega_{0}-\sigma / 2+c\right)$. But (8) holds also for every $\omega$ in $\omega_{0}-\sigma / 2<|\omega|<\omega_{0}+\sigma / 2$. To prove this, we use the $(1+r)$ th and the $(N+1+r)$ th equations of (3). Following the same steps as before, we find

$$
\begin{aligned}
\exp [j(\omega+r c) t] & =\sum_{i=1}^{2 N} H_{i}(\omega+r c) \\
& \times \sum_{n=-\infty}^{\infty} y_{i, 0}(t-n T) \exp (j n T \omega) \\
& =\sum_{i=1}^{2 N} H_{i}(\omega+r c) \sum_{n=-\infty}^{\infty} y_{i, 0}(t-n T) \\
& \times \exp [j n T(\omega+r c)]
\end{aligned}
$$

and

$$
\begin{aligned}
\exp \left[j\left(\omega+2 \omega_{0}+r c\right) t\right] & =\sum_{i=1}^{2 N} H_{i}\left(\omega+2 \omega_{0}+r c\right) \\
& \times \sum_{n=-\infty}^{\infty} y_{i, 0}(t-n T) \exp (j n T \omega) \\
& =\sum_{i=1}^{2 N} H_{i}\left(\omega+2 \omega_{0}+r c\right) \sum_{n=-\infty}^{\infty} y_{i, 0}(t-n T) \\
& \times \exp \left[j n T\left(\omega+2 \omega_{0}+r c\right)\right]
\end{aligned}
$$

in $\left(-\omega_{0}-\sigma / 2,-\omega_{0}-\sigma / 2+c\right)$; the last steps in (9a) and

(9b) being valid because

$$
\exp (j n T r c)=\exp (j n 2 \pi r)=1
$$

and, under generalized Brown's condition

$$
\exp \left[j n T\left(2 \omega_{0}+r c\right)\right]=\exp [j n 2 \pi(k+r)]=1 \text {. }
$$

The changes of variables $\omega+r c=\omega^{\prime}$ and $\omega+2 \omega_{0}+r c$ $=\omega^{\prime \prime}$ in (9a) and (9b) prove that (8) is valid in $-\omega_{0}-\sigma / 2$ $+r c<\omega<-\omega_{0}-\sigma / 2+(r+1) c$ and in $\omega_{0}-\sigma / 2+r c<\omega<$ $\omega_{0}-\sigma / 2+(r+1) c$, respectively. When $r$ varies between 0 and $N-1$, we cover the signal band (SB).

Using (8), we can write

$$
\begin{aligned}
x(t)= & \frac{1}{2 \pi} \int_{\mathrm{SB}} X(\omega) \exp (j \omega t) d \omega=\frac{1}{2 \pi} \int_{\mathrm{SB}} X(\omega) \\
& \times \sum_{i=1}^{2 N} H_{i}(\omega) \sum_{n=-\infty}^{\infty} y_{i, 0}(t-n T) \exp (j n T \omega) \\
& =\sum_{i=1}^{2 N} \sum_{n=-\infty}^{\infty} y_{i, 0}(t-n T) \frac{1}{2 \pi} \int_{\mathrm{SB}} X(\omega) H_{i}(\omega) \\
& \times \exp (j n T \omega) d \omega=\sum_{i=1}^{2 N} \sum_{n=-\infty}^{\infty} g_{i}(n T) y_{i, 0}(t-n T),
\end{aligned}
$$

where $g_{i}(t)$ is the output of the $i$ th linear time-invariant system. Expression (11) is a general interpolation formula.

\section{TO OBTAIN A SEPARATE INTERPOLATION}

A change of variable in (6) leads to

$y_{i, 0}(t)=\left(\frac{1}{c} \int_{-\sigma / 2}^{-\sigma / 2 * c} Y_{i}\left(\omega-\omega_{0}, t\right) \exp (j \omega t) d \omega\right) \exp \left(-j \omega_{0} t\right)$.

On the other hand, introducing the spectra of the complex envelops of the impulse responses

$$
\left.B_{h_{i}}(\omega)=2 H_{i}\left(\omega+\omega_{0}\right) d \omega+\omega_{0}\right), \quad i=1, \ldots, 2 N,
$$

where $u(\cdot)$ is the unit step function, it is clear that the system (3) can be written in the form

$$
\begin{aligned}
& \sum_{i=1}^{2 N} B_{h_{i}}^{*}[-(\omega+r c)] Y_{i}\left(\omega-\omega_{0}, t\right) \\
& \quad=2 \exp (j r c t), \quad r=0, \ldots, N-1 \\
& \sum_{i=1}^{2 N} B_{h_{i}}(\omega+r c) Y_{i}\left(\omega-\omega_{0}, t\right) \\
& \quad=2 \exp \left[j\left(2 \omega_{0}+r c\right) t\right], \quad r=0, \ldots, N-1
\end{aligned}
$$

in $-\sigma / 2<\omega<-\sigma / 2+c$. 
i.e.,

$$
\begin{aligned}
& B_{h_{i}}(\omega)=B_{h_{i}}^{*}(-\omega), \quad i=1, \ldots, N, \\
& B_{h_{i}}(\omega)=-B_{h_{i}}^{*}(-\omega), \quad i=N+1, \ldots, 2 N,
\end{aligned}
$$

(indices may be interchanged), and adding the $n$th and $(N+n)$ th equations, $n=1, \ldots, N$, we obtain

$$
\begin{aligned}
\sum_{i=1}^{N} B_{h_{i}}(\omega+r c) Y_{i}\left(\omega-\omega_{0}, t\right) \\
\quad=2 \exp (j r c t) \frac{\exp \left(j 2 \omega_{0} t\right)+1}{2}, r=0, \ldots, N-1
\end{aligned}
$$

and subtracting the $n$th from the $(N+n)$ th equations,

$$
\begin{aligned}
\sum_{i=N+1}^{2 N} B_{h i}(\omega+r c) Y_{i}\left(\omega-\omega_{0}, t\right) \\
\quad=2 \exp (j r c t) \frac{\exp \left(j 2 \omega_{0} t\right)-1}{2}, \quad r=0, \ldots, N-1 .
\end{aligned}
$$

Terms $\left[\exp \left(j 2 \omega_{0} t\right)+1\right] / 2$ and $\left[\exp \left(j 2 \omega_{0} t\right)-1\right] / 2$ give rise to, following (12), the factors

$$
\left[\exp \left(j 2 \omega_{0} t\right)+1\right] \exp \left(-j \omega_{0} t\right) / 2=\cos \omega_{0} t
$$

and

$$
\left[\exp \left(j 2 \omega_{0} t\right)-1\right] \exp \left(-j \omega_{0} t\right) / 2=j \sin \omega_{0} t
$$

in the $\left\{y_{i, 0}(t)\right\}_{i=1}^{N}$ and $\left\{y_{i, 0}(t)\right\}_{i=N+1}^{2 N}$, respectively. Then, $\left\{y_{i, 0}(t-n T)\right\}$ have only in-phase or quadrature components, because

$$
\sin _{\cos }^{\cos }\left[\omega_{0}(t-n T)\right]=(-1)^{n k} \cos \left(\omega_{0} t\right)
$$

using generalized Brown's condition.

Thus, with the assumptions (15a) and (15b), a separate interpolation is obtained. These assumptions are necessary, since they offer the only possibility to form the $\cos \omega_{0} t$ or $\sin \omega_{0} t$ factors in $\left\{y_{i, 0}(t)\right\}_{i=1}^{2 N}$.

The assumed conditions are equivalent to an identically zero quadrature component for the first $N$ impulse responses and an identically zero in-phase component for the last $N$ impulse responses. They are also equivalent to the following: $\operatorname{Re} H_{i}(\omega) u(\omega)$ and $\operatorname{Im} H_{i}(\omega) u(\omega)$ have to be symmetrical and antisymmetrical functions with respect to $\omega_{0}$, respectively, for $i=1, \ldots, N$, and just the contrary for $i=N+1, \ldots, 2 N$.

Strictly speaking, these conditions need to hold only in the signal band.

Systems (16a) and (16b) and formula (12) are easier to use than system (3) and formula (6) in these cases. Writing

$$
\begin{aligned}
& \sum_{i=1}^{N} B_{h_{i}}(\omega+r c) Z_{i}(\omega, t)=2 \exp (j r c t), \\
& r=0, \ldots, N-1, \\
& \sum_{i=N+1}^{2 N} B_{h_{i}}(\omega+r c) Z_{i}(\omega, t)=2 \exp (j r c t),
\end{aligned}
$$

$$
r=0, \ldots, N-1 \text {, }
$$

in $-\sigma / 2<\omega<-\sigma / 2+c$, and

$$
z_{i, 0}(t)=\frac{1}{c} \int_{-\sigma / 2}^{-\sigma / 2 * c} Z_{i}(\omega, t) \exp (j \omega t) d \omega
$$

the separate general interpolation formula is

$$
\begin{aligned}
x(t) & =\left(\sum_{i=1}^{N} \sum_{n=-\infty}^{\infty}(-1)^{k n} g_{i}(n T) z_{i, 0}(t-n T)\right) \cos \omega_{0} t \\
& -\left(\sum_{i=N+1}^{2 N} \sum_{n=-\infty}^{\infty}(-1)^{k n} g_{i}(n T)\left[-j z_{i, 0}(t-n T)\right) \sin \omega_{0} t .\right.
\end{aligned}
$$

Note that, since

$$
\begin{aligned}
& B_{h_{i}}(\omega)=P_{h_{i}}(\omega), \quad i=1, \ldots, N, \\
& B_{h_{i}}(\omega)=j Q_{h_{i}}(\omega), \quad i=N+1, \ldots, 2 N,
\end{aligned}
$$

where $P_{h_{i}}(\omega), Q_{h_{i}}(\omega)$, indicate the spectra of the in-phase and quadrature components of $h_{i}(t)$, respectively (therefore, they are Hermitian functions), $\left\{z_{i, 0}(t)\right\}_{i=1}^{N}$ and $\left\{z_{i, 0}(t)\right\}_{i=N+1}^{2 N}$ will be real and purely imaginary functions, respectively. Then, (21) is a real expression.

\section{AN ILLUSTRATIVE EXAMPLE}

Let us select $N=2$

$$
\begin{aligned}
H_{i}(\omega) & =\Pi\left(\frac{\omega-\omega_{0}}{\sigma}\right)+\Pi\left(\frac{\omega+\omega_{0}}{\sigma}\right), \\
H_{2}(\omega) & =j\left[\left(\omega-\omega_{0}\right) \Pi\left(\frac{\omega-\omega_{0}}{\sigma}\right)+\left(\omega+\omega_{0}\right) \Pi\left(\frac{\omega+\omega_{0}}{\sigma}\right)\right], \\
H_{3}(\omega) & =\left[\Pi\left(\frac{\omega-\omega_{0}}{\sigma}\right)+\Pi\left(\frac{\omega+\omega_{0}}{\sigma}\right)\right] \exp \left(-j \omega \pi / 2 \omega_{0}\right) \\
H_{4}(\omega) & =j\left[\left(\omega-\omega_{0}\right) \Pi\left(\frac{\omega-\omega_{0}}{\sigma}\right)+\left(\omega+\omega_{0}\right) \Pi\left(\frac{\omega+\omega_{0}}{\sigma}\right)\right] \\
& \times \exp \left(-j \omega \pi / 2 \omega_{0}\right),
\end{aligned}
$$

that imply

$$
\begin{aligned}
& g_{1}(t)=x(t), \\
& g_{2}(t)=\left[d p_{x}(t) / d t\right] \cos \omega_{0} t-\left[d q_{x}(t) / d t\right] \sin \omega_{0} t, \\
& g_{3}(t)=x\left(t-\pi / 2 \omega_{0}\right), \\
& g_{4}(t)=g_{2}\left(t-\pi / 2 \omega_{0}\right),
\end{aligned}
$$

and spectra of complex envelopes

$$
\begin{aligned}
& B_{h_{1}}(\omega)=2 \Pi(\omega / \sigma), \\
& B_{h_{2}}(\omega)=2 j \omega \Pi(\omega / \sigma), \\
& B_{h_{3}}(\omega)=-2 j \Pi(\omega / \sigma) \exp \left(-j \omega \pi / 2 \omega_{0}\right), \\
& B_{h_{4}}(\omega)=2 \omega \Pi(\omega / \sigma) \exp \left(-j \omega \pi / 2 \omega_{0}\right),
\end{aligned}
$$

that satisfy the conditions for a separate interpolation. Applying (19a) and (19b), we arrive at $(c=\sigma / 2)$

$Z_{1}(\omega, t)+j \omega Z_{2}(\omega, t)=1$ 


$$
\begin{aligned}
& Z_{1}(\omega, t)+j(\omega+\sigma / 2) Z_{2}(\omega, t)=\exp (j \sigma t / 2) \\
& -j \exp \left(-j \omega \pi / 2 \omega_{0}\right) Z_{3}(\omega, t)+\omega \exp \left(-j \omega \pi / 2 \omega_{0}\right) Z_{4}(\omega, t) \\
& =1-j \exp \left[-j(\omega+\sigma / 2) \pi / 2 \omega_{0}\right] Z_{3}(\omega, t)+(\omega+\sigma / 2) \\
& \quad \times \exp \left[-j(\omega+\sigma / 2) \pi / 2 \omega_{0}\right] Z_{4}(\omega, t)=\exp (j \sigma t / 2) \\
& \text { in }-\sigma / 2<\omega<0 . \text { From }(26), \\
& \quad Z_{1}(\omega, t)=1-(2 \omega / \sigma)[\exp (j \sigma t / 2)-1], \\
& \quad Z_{2}(\omega, t)=(2 / j \sigma)[\exp (j \sigma t / 2)-1],
\end{aligned}
$$

and, from (27),

$$
\begin{aligned}
Z_{3}(\omega, t) & =j \exp \left(j \omega \pi / 2 \omega_{0}\right) \\
& \times\left(1-(2 \omega / \sigma)\left\{\exp \left[j \sigma\left(t+\pi / 2 \omega_{0}\right) / 2\right]-1\right\}\right), \\
Z_{4}(\omega, t)= & (2 / \sigma) \exp \left(j \omega \pi / 2 \omega_{0}\right) \\
& \times\left\{\exp \left[j \sigma\left(t+\pi / 2 \omega_{0}\right) / 2\right]-1\right\} .
\end{aligned}
$$

Applying (20), we obtain

$$
\begin{aligned}
& z_{1,0}(t)=16 \sin ^{2}(\sigma t / 4) / \sigma^{2} t^{2}, \\
& z_{2,0}(t)=16 \sin ^{2}(\sigma t / 4) / \sigma^{2} t, \\
& -j z_{3,0}(t)=16 \sin ^{2}\left[\sigma\left(t+\pi / 2 \omega_{0}\right) / 4\right] / \sigma^{2}\left(t+\pi / 2 \omega_{0}\right)^{2}, \\
& -j z_{4,0}(t)=16 \sin ^{2}\left[\sigma\left(t+\pi / 2 \omega_{0}\right) / 4\right] / \sigma^{2}\left(t+\pi / 2 \omega_{0}\right) .
\end{aligned}
$$

Inserting (30a) - (30d) in (21), we will obtain the corresponding separate interpolation formula.

\section{WHEN GENERALIZED BROWN'S CONDITION IS NOT SATISIFIED}

When $\omega_{0} \neq k \sigma / 2 N$, it is possible to maintain the previous formulation in each of the two ways proposed for the case $N=1$ (Brown's condition). ${ }^{5-7}$ We will review briefly these ways, adapting them to the general case.

If $\omega_{0}$ must be maintained in formula (21) (because there is some symmetry with respect to $\omega_{0}$, or because $\omega_{0}$ is a carrier frequency), we will use the smallest $\sigma^{\prime}>\sigma$ such that $\omega_{0}=k \sigma^{\prime} / 2 N$, i.e.,

$$
\sigma^{\prime}=2 N \omega_{0} / E\left[2 N \omega_{0} / \sigma\right],
$$

where $E[\cdot]$ indicates the greatest integer function. The corresponding new sampling rate will be

$$
1 / T^{\prime}=\sigma^{\prime} / 2 \pi N=\omega_{0} / \pi E\left[2 N \omega_{0} / \sigma\right] .
$$

$\sigma^{\prime}$ and $T^{\prime}$ will take the places of $\sigma$ and $T$, respectively, in (21).

In a general situation, since band limitation is not a physical possibility, $\omega_{0}$ need not have the strict sense of center angular frequency; then, we can search for the minimum sampling rate without maintaining $\omega_{0}$. The corresponding new bandwidth $\sigma^{\prime \prime}$ is obtained by dividing $2 N \omega_{0}+\sigma$ in the maximum number of equal segments of width $\sigma^{\prime \prime}>\sigma$; then

$$
\sigma^{\prime \prime}=\left(2 N \omega_{0}+\sigma\right) / E\left[\left(2 N \omega_{0}+\sigma\right) / \sigma\right]
$$

and we have a sampling rate

$$
\begin{aligned}
1 / T^{\prime \prime} & =\sigma^{\prime \prime} / 2 \pi N=\left(2 N \omega_{0}+\sigma\right) / 2 \pi N E \\
& \times\left[\left(2 N \omega_{0}+\sigma\right) / \sigma\right]<1 / T^{\prime}=\sigma^{\prime} / 2 \pi N \\
& =\left(2 N \omega_{0}+\sigma^{\prime}\right) / 2 \pi N E\left[\left(2 N \omega_{0}+\sigma\right) / \sigma\right] .
\end{aligned}
$$

The corresponding new angular center frequency is

$$
\omega_{0}^{\prime \prime}=\omega_{0}+\sigma / 2-\sigma^{\prime \prime} / 2 \text {. }
$$

$\sigma^{\prime \prime}, T^{\prime \prime}$, and $\omega_{0}^{\prime \prime}$ will take the places of $\sigma, T$, and $\omega_{0}$, respectively, in (21).

$\sigma^{\prime \prime}$ is the best solution in this second situation, because increasing the spectral interval to be segmented does not allow a sampling rate $\sigma^{\prime \prime \prime}<\sigma^{\prime \prime}$. To prove this, we can use the following argument: If the interval were increased and we look for $\sigma^{m}<\sigma^{\prime \prime}$, the number of segments would increase also; it would be

$$
\left(2 N \omega_{0}^{m}+\sigma^{\prime \prime}\right) / \sigma^{m \prime} \geqslant E\left[\left(2 N \omega_{0}+\sigma\right) / \sigma\right],
$$

where $\omega_{0}^{\prime \prime \prime}$ would be the new center angular frequency. Equation (36) would imply

$$
\omega_{0}^{\prime \prime \prime}>\omega_{0}
$$

It would be also necessary that the $N$ segments of width $\sigma^{\prime \prime \prime} / N$ disposed around $\pm \omega_{0}^{\prime \prime \prime}$ cover the signal band; this implies

$$
\omega_{0}^{\prime \prime \prime}-\sigma^{\prime \prime \prime} / 2 \leqslant \omega_{0}-\sigma / 2,
$$

i.e.,

$$
\sigma^{\prime \prime \prime} \geq \sigma+2\left(\omega_{0}^{m \prime}-\omega_{0}\right) .
$$

Introducing the minimum value of $\sigma^{\prime \prime \prime}$ according to (39) in (36), we obtain

$$
\frac{2 N \omega_{0}^{\prime \prime}+2\left(\omega_{0}^{\prime \prime \prime}-\omega_{0}\right)}{\sigma+2\left(\omega_{0}^{\prime \prime \prime}-\omega_{0}\right)} \geqslant E\left[\frac{2 N \omega_{0}}{\sigma}+1\right]
$$

and this is not possible when $\omega_{0}^{\prime \prime \prime}>\omega_{0}$ and $\omega_{0}>\sigma / 2$. This impossibility is maintained for greater values of $\sigma^{\prime \prime \prime}$.

\section{CONCLUSIONS}

We have shown that it is possible to obtain separate interpolation of the components of a band-limited bandpass signal from samples of $2 \mathrm{~N}$ linear functionals of it, taken at a rate equal to $1 / 2 \mathrm{~N}$ times the Nyquist rate, with only three restrictions:

(a) the transfer functions corresponding to the $2 \mathrm{~N}$ linear functionals have to provide a solution for the system (3) in $-\omega_{0}-\sigma / 2<\omega<-\omega_{0}-\sigma / 2+\sigma / N$;

(b) the center angular frequency and the bandwidth of the signal will be related by $\omega_{0}=k \sigma / 2 N$, where $k$ is an integer (generalized Brown's condition);

(c) the impulse responses of $N$ systems have identically zero quadrature components, and the impulse responses of the other $N$ systems have identically zero inphase components.

Some known results (corresponding to $N=1$ ) are particular cases of this general method.

This formulation is of interest because it allows reductions of individual sampling rates (maintaining $\mathrm{Ny}-$ quist rate on the average) and the use of a minimum number of interpolating filters in equivalent lowpass processing (or, equivalently, a minimum number of "frequency-continuous" bandpass interpolating filters). 
When generalized Brown's condition is not satisfied, we can apply the described procedure with a minimum sampling rate $1 / T^{\prime}=\sigma^{\prime} / 2 \pi N=\omega_{0} / \pi E\left[2 N \omega_{0} / \sigma\right]$ maintaining the center angular frequency, or with a minimum sampling rate $1 / T^{n}=\sigma^{n} / 2 \pi N=\left(2 N \omega_{0}+\sigma\right) / 2 \pi N E\left[\left(2 N \omega_{0}+\sigma\right) / \sigma\right]$ without maintaining $\omega_{0}$.

\section{ACKNOWLEDGMENTS}

The authors wish to thank Professor Athanasios Papoulis for focusing their attention to his Generalized Sampling Expansion, and to Professor John L. Brown, Jr., for many helpful comments, suggestions, and discussions.

${ }^{1} \mathrm{~J}$. L. Brown, Jr, "On Quadrature-Sampling of Bandpass Signals," IEEE Trans. Aerosp. Electron. Syst. AES-15, 366-371 (1979).

${ }^{2}$ L. E. Franks, Signal Theory ( Prentice-Hall, Englewood Cliffs, 1969).

${ }^{3}$ S. Goldman, Information Theory (Prentice-Hall, New York, 1953 ).
"O. D. Grace and S. P. Pitt, "Quadrature Sampling of HighFrequency Waveforms," J. Acoust. Soc. Am. 44, 1453-1454 (1968).

5J. L. Brown, Jr., "A Simplified Approach to Optimum Quadrature Sampling," J. Acoust. Soc. Am. 67, 1659-1662 (1980).

${ }^{6}$ A. R. Figueiras-Vidal, J. B. Marino-Acebal, M. A. Lagunas-Hernandez, and R. Garcia-Gomez, "Some New Results in Sampling Deterministic Signals," Proc. First European Conf. Signal Proc., 197-200, Lausanne, Switzerland (1980).

${ }^{7}$ A. R. Figueiras-Vidal, "Sampling Bandpass Signals," IEEE Trans. Aerosp. Electron. Sys. AES-17, 288-295 (1981).

${ }^{8}$ A. Papoulis, "New Results in Sampling Theory," Hawall Int. Conf. Syst. Sci. (1968).

${ }^{9} \mathrm{~A}$. Papoulis, Systems and Transforms with Applications in Optics (McGraw-Hill, New York, 1968).

${ }^{10}$ A. Papoulis, "Generalized Sampling Expansion," IEEE Trans. Circuits Syst. CAS-24, 652-654 (1977).

${ }^{11} \mathrm{~J}$. L. Brown, Jr., "Generalized Sampling of Bandpass Signals" ( abstract), Proc. IEEE Int. Symp. on Inf. Theory, 155, Grignano, Italy (1979).

${ }^{12} J$. L. Brown, Jr., "Multi-Channel Sampling of Lowpass Signals," IEEE Trans. Círcuit Syst. CAS-28, 101-106 (1981).

${ }^{13} \mathrm{~J}$. L. Brown, Jr., "Sampling Rate Reduction in Multi-Channel Processing of Bandpass Signals," J. Acoust. Soc. Am. (submitted for publication). 